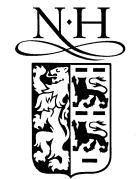

ELSEVIER

\title{
Colliding-pulse mode-locked semiconductor laser with a multimode-interference waveguide amplifier
}

\author{
Chih-Chiarng Chen, Yean-Woei Kiang * , C.C. Yang \\ Department of Electrical Engineering, Graduate Institute of Communication Engineering, and Graduate Institute of Electro-Optical \\ Engineering, National Taiwan University, 1, Roosevelt Road, Sec. 4, Taipei, Taiwan
}

Received 28 October 1999; received in revised form 4 February 2000; accepted 22 February 2000

\begin{abstract}
We demonstrate numerically a novel colliding-pulse mode-locking (CPM) technique for semiconductor laser without using a saturable absorber. A multimode-interference (MMI) semiconductor optical amplifier (SOA) is used as the gain medium and the mode locker. Efficient pulse compression and stabilization of the two counter-propagating pulses are achieved based on the nonlinear-coupling effect, assisted with pulse collision, in the MMI SOA. Two initially different pulses tend to adjust each other, resulting in symmetric collision of two identical pulses. Such efficient pulse compression and stabilization mechanisms should lead to simple implementation of CPM semiconductor lasers. (C) 2000 Elsevier Science B.V. All rights reserved.
\end{abstract}

Colliding-pulse mode-locking (CPM) was the key technique for generating femtosecond pulses in dye lasers, which were the major tools for ultra-fast research in the 1980s. It was later applied to semiconductor lasers, leading to femtosecond pulses after external-cavity dispersion compensation [1,2]. Basically, a CPM laser includes a saturable absorber where the counter-propagating pulses collide. In collision, the two pulses enhance the pulse compression effect of each other through power dependent ab-

\footnotetext{
* Corresponding author. Tel.: +886-2-2363-5251 ext. 438; fax: +886-2-2365-2637; e-mail: ywkiang@ew.ee.ntu.edu.tw
}

sorption $[3,4]$. Such a process includes an implication that the two colliding pulses tend to adjust themselves such that they enter the saturable absorber from the two ends at the same time and hence collide at the center of the saturable absorber. This adjustment is attributed to the fact that the earlier-arriving pulse saturates the absorption for effectively enhancing the strength of the leading edge of the later-arriving pulse. This equivalently results in the advance of the later-arriving pulse and the delay of the earlierarriving pulse. Such an adjustment is important for stabilizing the mode-locked pulses.

Such a stabilization mechanism does not exist if the saturable absorber is replaced by a saturable gain 
medium. Therefore, a saturable absorber is required in a conventional CPM laser for generating stable pulses. However, this requirement represents more loss and complexity for the laser. If a gain medium of a properly designed configuration can be used to replace the saturable absorber with the same function kept, CPM lasers can be simplified. In this letter, we propose a novel configuration for CPM semiconductor laser without the need of a saturable absorber. It is based on the nonlinear coupling effect in a multimode-interference (MMI) semiconductor optical amplifier (SOA) or a directional-coupler amplifier. We will use an MMI SOA structure to numerically demonstrate the feasibility of CPM.

The layout of such a laser system is shown in Fig. 1 , in which the MMI SOA with four input/output ports is placed at the center of the resonance cavity. Assume that there exist two waveguide modes in the MMI SOA, which is a ridge-loading waveguide. The input/output ports are assumed to be passive (can be implemented through quantum well intermixing techniques). Laser signal oscillates between mirrors M1 and M2 and outputs are obtained from the reflection of mirrors M3 and M4. For convenience, the two ports corresponding to mirrors M1 and M2 will be called the bar-ports and those corresponding to M3 and M4 are called the cross-ports. For numerical simplicity, we neglect all the reflections from the connections between the MMI SOA and the four passive waveguide ports and from the interfaces between the passive waveguide ports and the air. The reflectivities of the four mirrors are assumed to be $100 \%$. Therefore, we need not consider the boundary conditions in the $z$-direction. It is also assumed that the two external-cavities are quite long so that gain can essentially recover from saturation when the

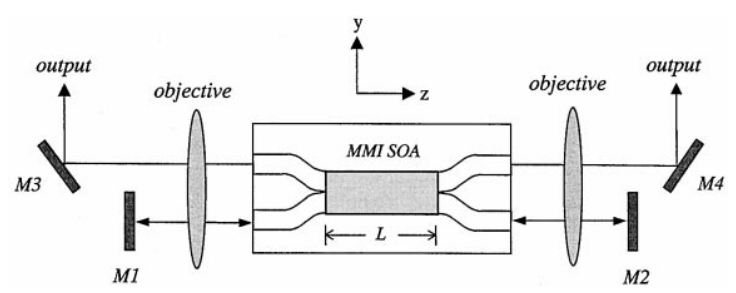

Fig. 1. Layout of the CPM semiconductor laser. The MMI SOA is placed at the center of the cavity, which is formed with mirrors M1 and M2. Counter-propagating pulses oscillate through the two bar-ports. oscillating pulses return to the MMI SOA. Typically, a $15-\mathrm{cm}$ long external-cavity length is sufficient. In addition, the length of the MMI SOA is assumed to be one-half beat length such that a weak signal incident from one side of the lateral dimension emerges from the other side (cross-port). As shown in Fig. 1, the pulses enter from the two ends of the MMI SOA on the same side of the lateral dimension, i.e., bar-ports.

To simulate the collision of pulses in the MMI SOA, we expanded the TE polarized electric field into two waveguide modes (modes 1 and 2), each of which propagates in both $+z$ and $-z$ directions. The corresponding four slowly-varying wave amplitudes, $A_{1}^{+}(z, t), A_{2}^{+}(z, t), A_{1}^{-}(z, t)$ and $A_{2}^{-}(z, t)$, satisfy the following equations:

$$
\begin{aligned}
&\left(\frac{1}{\nu_{\mathrm{g}}} \frac{\partial}{\partial t}+\frac{\partial}{\partial z}\right) A_{1(2)}^{+}(z, t) \frac{1}{2}(1+\mathrm{j} \alpha) \bar{g}_{1(2)}(z, t) A_{1(2)}^{+}(z, t) \\
&+\frac{1}{2}(1+\mathrm{j} \alpha) \bar{g}_{x}(z, t) A_{2(1)}^{+}(z, t) \mathrm{e}^{ \pm \mathrm{j} \Delta \beta z} \\
&-\frac{1}{2} \rho A_{1(2)}^{+}(z, t) \\
&\left.\frac{1}{\nu_{\mathrm{g}}} \frac{\partial}{\partial t}-\frac{\partial}{\partial z}\right) A_{1(2)}^{-}(z, t) \\
&=\frac{1}{2}(1+\mathrm{j} \alpha) \bar{g}_{1(2)}(z, t) A_{1(2)}^{-}(z, t) \\
& \quad+\frac{1}{2}(1+\mathrm{j} \alpha) \bar{g}_{x}(z, t) A_{2(1)}^{-}(z, t) \mathrm{e}^{\mp \mathrm{j} \Delta \beta(z-L)} \\
& \quad-\frac{1}{2} \rho A_{1(2)}^{-}(z, t)
\end{aligned}
$$

with $\bar{g}_{1(2)}(z, t) \equiv \int_{-\infty}^{\infty} g(y, z, t) F_{1(2)}^{2}(y) \mathrm{d} y \quad$ and $\bar{g}_{x}(z, t) \equiv \int_{-\infty}^{\infty} g(y, z, t) F_{1}(y) F_{2}(y) \mathrm{d} y$.

Here, $g(y, z, t)$ is the gain constant distribution and $F_{1(2)}(y)$ is the normalized lateral pattern of mode 1(2) with a sinusoidal pattern inside the ridge region and exponential decay outside. Parameter $\Delta \beta$ $=\beta_{1}-\beta_{2}$ is the difference between the propagation constants of the two modes, $\nu_{\mathrm{g}}$ is the group velocity, $\rho$ is the loss constant, $\alpha(=6)$ is the linewidth enhancement factor, and $L(=665 \mu \mathrm{m})$ is the length 
of the MMI SOA. Note that the variation along the epitaxial growth direction $(x)$ has been averaged out. Also, the gain constant $g(y, z, t)$ is related to the optical intensity $|E(y, z, t)|^{2}$ through the equation

$$
\begin{aligned}
& \frac{\partial g(y, z, t)}{\partial t} \\
& \quad=\frac{g_{0}-g(y, z, t)}{\tau_{\mathrm{c}}}-\frac{|E(y, z, t)|^{2}}{E_{\text {sat }} / \sigma} g(y, z, t)
\end{aligned}
$$

where $g_{0}$ is the small-signal gain constant, $\tau_{\mathrm{c}}(=1$ ns) is the carrier lifetime, $\sigma(=8 \times 0.43 \mu \mathrm{m})$ is the effective cross-sectional area of the waveguide, and $E_{\text {sat }}(=5 \mathrm{pJ})$ is the saturation energy. Note that we are concerned with the effects of carrier dynamics on pulse evolution in the time range from hundreds to a few ps over a lateral dimension of around $8 \mu \mathrm{m}$. Within such a short time range, the effects of carrier diffusion can be neglected [5]. For instance, a lateral diffusion time constant at $1.4 \mathrm{~ns}$ was estimated for a scale size of $1.5 \mu \mathrm{m}$ in a GaAs/AlGaAs structure [6]. In our case, with the $8-\mu \mathrm{m}$ concerned lateral dimension, the diffusion time constant would exceed $10 \mathrm{~ns}$, which is much larger than the pulse width and carrier lifetime. Therefore, the diffusion factor is neglected in Eq. (3). However, under other conditions the diffusion effect may deserve further investigation.

For numerical simulation, the time-domain traveling-wave model [7] was adopted. However, instead

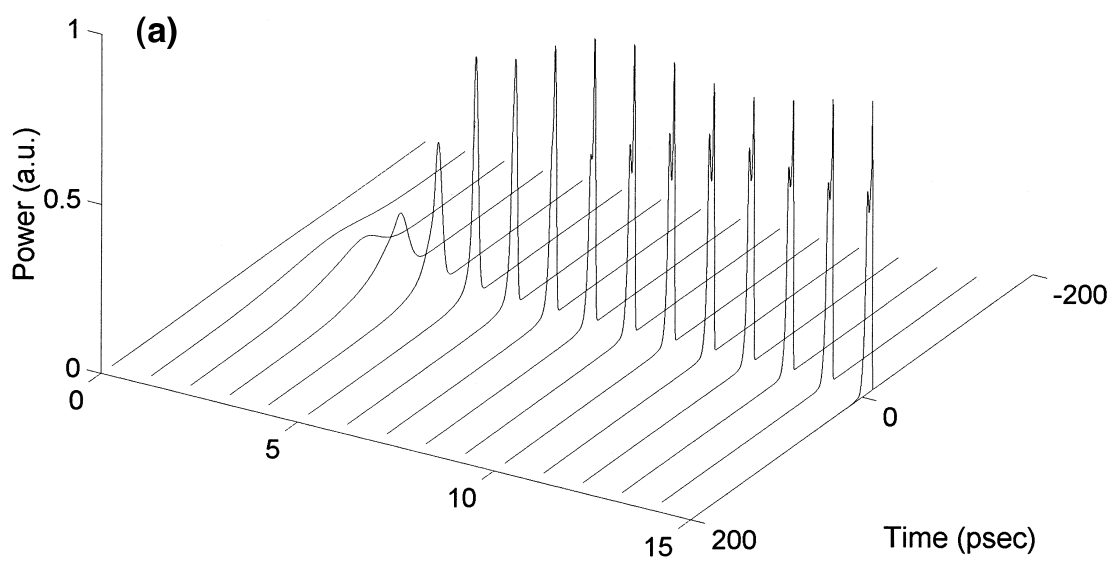

Round Trips

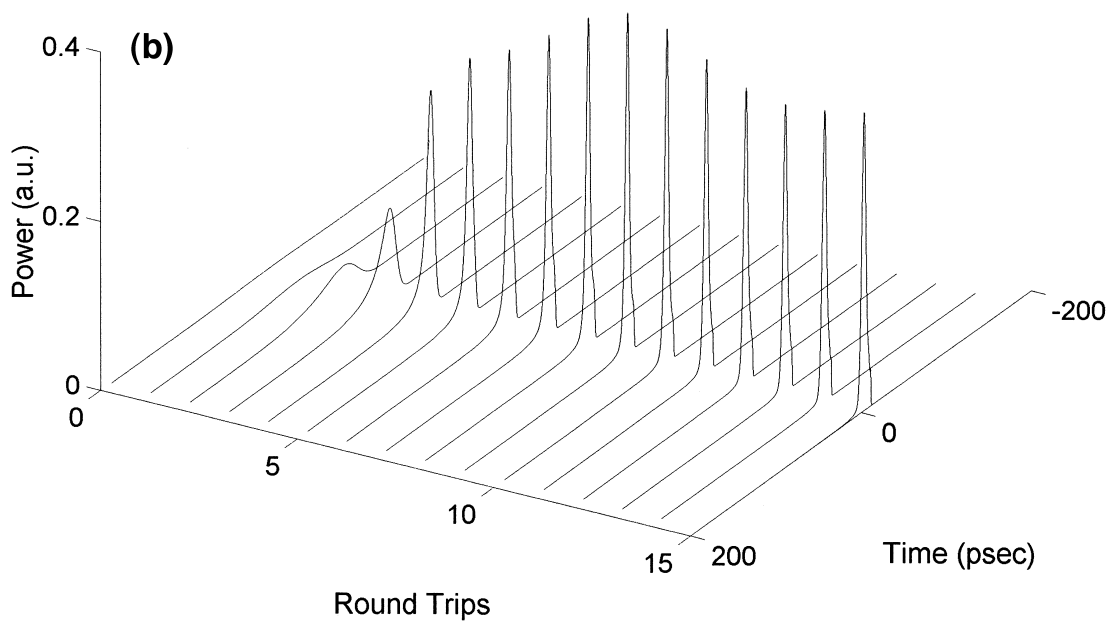

Fig. 2. Evolution of one of the counter-propagating pulses with (a) for the cross-port and (b) for the bar-port. 
of applying the first-order finite-difference approximation to the partial derivatives [7], we analytically solved the differential equations in each small segment $\Delta z$ along the axis of the MMI SOA.

Fig. 2(a) and Fig. 2(b) show the pulse evolution emerging from the cross- and bar-port, respectively. It was assumed that two initially identical long pulse of width $100 \mathrm{ps}$ and energy $0.04 E_{\text {sat }}$ entered the MMI SOA simultaneously. Therefore, the evolutions of the two counter-propagating pulses are symmetric. The small-signal gain factor and internal loss coefficient in the MMI SOA were set at $20 \mathrm{~dB}$ and 19 $\mathrm{cm}^{-1}$, respectively. One can see that pulses from both cross- and bar-port are compressed along oscillation. After several round-trips, the pulses approach their asymptotic shapes. Fig. 3 shows the evolutions of the FWHM pulse width and the pulse peak position in the cross- (C) and bar-port (B). One can see that after several round-trips, the pulse width and temporal position reach almost steady-state values. This is an indication that mode locking is feasible in such a laser system.

It is recalled that like a directional-coupler SOA, an MMI SOA with one-half beat length can result in the nonlinear coupling and pulse breakup effects [8]. In other words, because of gain saturation, different temporal portions of a pulse will emerge from either the bar- or cross-port. In our simulation, we find that a fraction of the central portion (near the peak) of the pulse emerges from the bar-port after a single pulse

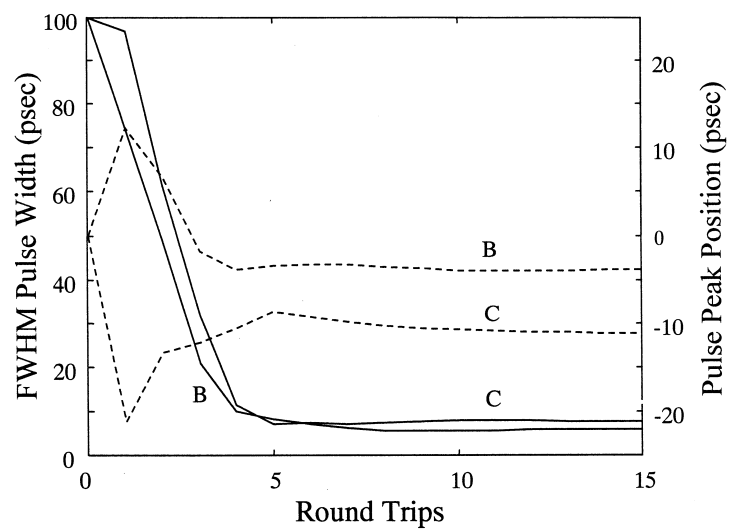

Fig. 3. Evolutions of the FWHM pulse widths (solid curves) and pulse peak positions (dashed curves) of the bar-port (labeled B) and cross-port (labeled C) pulses. propagating through the MMI SOA. This bar-port pulse has a width shorter than the input pulse. Such a pulse compression mechanism in a directional-coupler SOA [9] or an MMI SOA [10] has been proposed for passive mode locking of a semiconductor laser with a ring-cavity configuration. Here, it is discovered that with collision of two counterpropagating pulses, as shown in Fig. 1, the pulse compression is more efficient. Therefore, with pulse oscillating between mirrors M1 and M2, pulses are continuously narrowed. Such a mechanism is expected to result in effective CPM. Although groupvelocity dispersion was not considered in our simulation, the pulse width can reach a steady state. This is attributed to the fact that both the clipping effect (due to linear coupling) on the pulse leading side and the effective intensity depression (due to gain saturation) on the trailing side diminish gradually as pulse width becomes narrower. The pulse position reaches its steady state value because the aforementioned clipping effect on the leading side stops the pulse advance, which is due to gain saturation. The mutual enhancement of pulse compression is attributed to the fact that the coexistence of the counter-propagating pulses can enhance nonlinear coupling in the MMI SOA. We also tried the case that two counterpropagating pulses entered the MMI SOA on different sides of the lateral dimension and found that the mutual enhancement of pulse compression did not exist.

As mentioned before, a pulse position stabilization mechanism exists in the saturable absorber of a conventional CPM laser. Such a mechanism may not exist in a single-waveguide-mode SOA. However, it exists in the MMI SOA, used for our CPM laser. The four curves in Fig. 4 show the bar-port output evolutions of the FWHM pulse widths and pulse peak positions of the two counter-propagating pulses, which enter the MMI SOA at different times by 165 ps. One can see that after several round-trips, the pulse widths and peak positions approach their asymptotic values. Of particular interest is that the earlier-arriving pulse has been delayed by 129 ps and the later-arriving pulse has been advanced by $36 \mathrm{ps}$, leading to the result that two pulses collide at the center of the MMI SOA. This is the mechanism for pulse position stabilization, similar to a conventional CPM laser. 


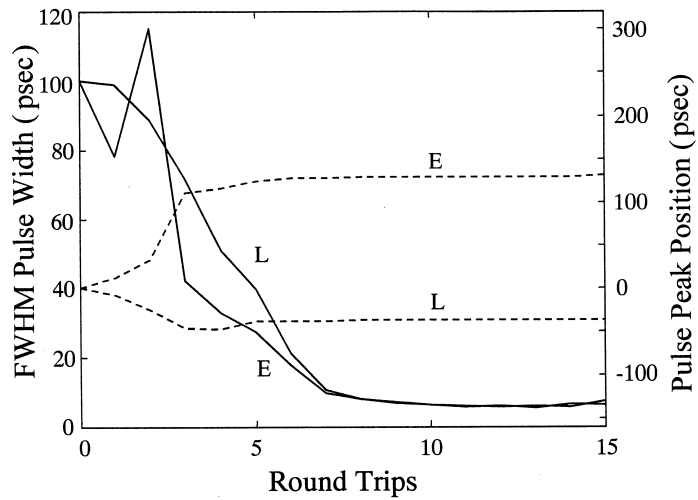

Fig. 4. Evolutions of the FWHM pulse widths (solid curves) and pulse peak positions (dashed curves) of the bar-port outputs for the earlier-arriving pulse (labeled E) and later-arriving pulse (labeled L).

Shown in Fig. 5 are the bar-port output evolutions of the pulse energies and the FWHM pulse widths of two counter-propagating pulses, which enter the MMI SOA simultaneously. Here, the two initial pulses have the same width (100 ps), but different pulse energies (one at $0.04 E_{\mathrm{sat}}$ and the other at $0.01 E_{\mathrm{sat}}$ ). It can be seen that after several round-trips, the pulse widths and energies approach their asymptotic values. Note that the energies of the two pulses become equal after one round-trip. This again leads to symmetric collision. In other simulations, we also find that the collision between two pulses of different widths tends to equalize their widths along oscilla-

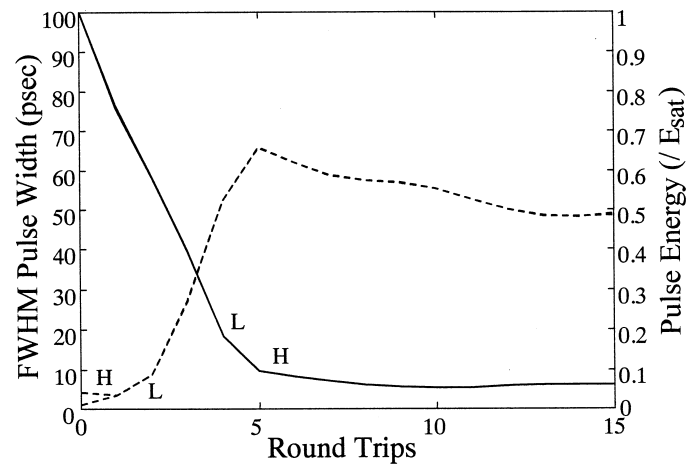

Fig. 5. Evolutions of the FWHM pulse widths (solid curves) and pulse energies (dashed curves) of the bar-port outputs for the low-energy pulse (labeled L) and high-energy pulse (labeled H). tion. The adjustments of pulse position and width are attributed to the fact that both pulses in the bar-port would receive the most gain when the system becomes symmetric. The detailed explanations will be presented in another publication.

Note that our theoretical model does not include the effects of gain dispersion, which are important for subpicosecond signals. The effective groupvelocity dispersion (GVD) due to gain dispersion may cause pulse broadening. To evaluate this effect, one can define a dispersion length $L_{\mathrm{D}}[11]$ as

$L_{\mathrm{D}}=\frac{0.36 \tau_{\mathrm{p}}^{2}}{\alpha g_{0} T_{2}^{2}}=\frac{0.36 \tau_{\mathrm{p}}^{2}}{\beta_{2}^{\text {eff }}}$

where $T_{2}$ is the gain relaxation time, $\alpha$ is the linewidth enhancement factor, $g_{0}$ is the unsaturated gain constant, $\tau_{\mathrm{p}}$ is the FWHM pulsewidth, and $\beta_{2}^{\text {eff }}=\alpha g_{0} T_{2}^{2}$ is the effective GVD parameter introduced in a manner similar to that of optical fiber. For $\alpha=6, T_{2}=0.1 \mathrm{ps}$, and $g_{0}=10000 \mathrm{~m}^{-1}, \beta_{2}^{\text {eff }}$ is $600 \mathrm{ps}^{2} / \mathrm{m}$. The effect of GVD due to gain dispersion becomes important for an amplifier of length $L \geq L_{\mathrm{D}}$. In our simulations, the smallest FWHM

(a)
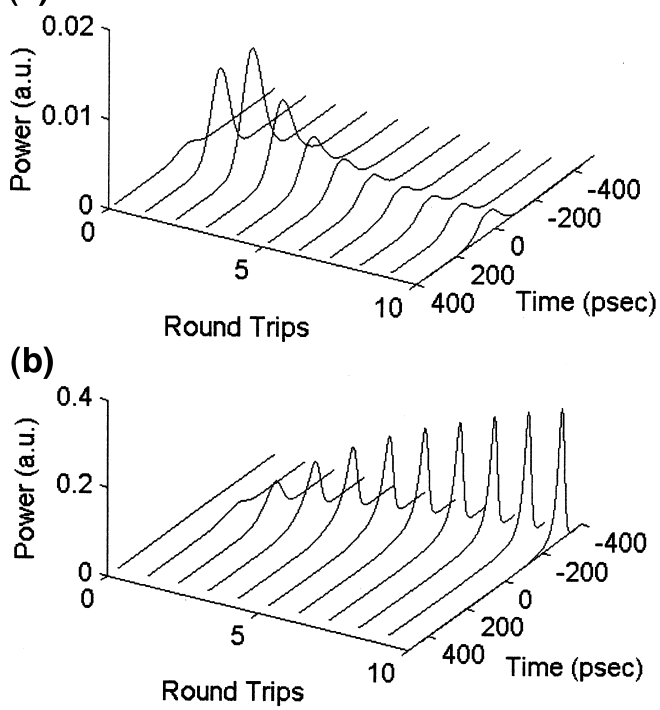

Fig. 6. Evolutions of the counter-propagating pulses when the MMI SOA is replaced by a single-waveguide-mode SOA for later-arriving pulse (a) and earlier-arriving pulse (b). 
pulsewidth is about 5 ps. Hence, the corresponding dispersion length is $L_{\mathrm{D}} \cong 1.5 \mathrm{~cm}$, which is much larger than the length $(665 \mu \mathrm{m})$ of our MMI SOA. Therefore, this dispersion effect is negligible. Nevertheless, the gain-induced GVD would be important for the subpicosecond case and its effects deserve further study.

To assure that the MMI configuration is required for the pulse stabilization mechanism, Fig. 6(a) and Fig. 6(b) demonstrate pulse evolution when the MMI SOA is replaced by a single-waveguide-mode SOA and the two counter-propagating pulses enter the SOA with one delayed by $75 \mathrm{ps}$ from the other. One can see that the delayed one diminishes (a) and the other grows and becomes narrower (b). The growing one advances during oscillation. However, it does not seem to reach a steady state.

In summary, we have numerically demonstrated the feasibility of a novel CPM semiconductor laser without using a saturable absorber. The nonlinear coupling effect, assisted with pulse collision, in an MMI SOA led to efficient pulse compression and stabilization. Two initially different pulses, which might come from noise bursts in a real laser system, would evolve into symmetric collision of two identical pulses. These results guarantee the feasibility of a CPM laser.

\section{Acknowledgements}

This research was supported by the grants NSC 88-2215-E-002-016, NSC 88-2112-M-002-004, NSC 88-2215-E-002-012, and NSC 88-2215-E-002-040 from National Science Council, the Republic of China.

\section{References}

[1] Y.K. Chen, M.C. Wu, T. Tanbun-Ek, R.A. Logan, M.A. Chin, Appl. Phys. Lett. 58 (1991) 1253.

[2] C.F. Lin, C.L. Tang, Appl. Phys. Lett. 62 (1993) 1053.

[3] D.J. Jones, L.M. Zhang, J.E. Carroll, D.D. Marcenac, IEEE J. Quantum Electron. 31 (1995) 1051.

[4] W. Chen, J. Zhu, S. Liu, IEEE J. Quantum Electron. 31 (1995) 1663.

[5] G.P. Agrawal, A. Olsson, IEEE J. Quantum Electron. 25 (1989) 2297.

[6] P. LiKamWa, A. Miller, C.B. Park, J.S. Roberts, P.N. Robson, Appl. Phys. Lett. 57 (1990) 1846.

[7] L.M. Zhang, S.F. Yu, M.C. Nowel, D.D. Marcenac, J.E. Carroll, R.G.S. Plumb, IEEE J. Quantum Electron. 30 (1994) 1389.

[8] S. Trillo, S. Wabnitz, J.M. Soto-Crespo, E.M. Wright, IEEE J. Quantum Electron. 27 (1991) 410.

[9] C.W. Hsu, C.C. Yang, Opt. Lett. 21 (1996) 878.

[10] J.C. Lai, Y.W. Kiang, C.C. Yang, IEEE J. Quantum Electron. 35 (1999) 1630.

[11] G.P. Agrawal, IEEE J. Quantum Electron. 27 (1991) 1843. 